\title{
Maximizing the quality factor to mode volume ratio for ultra-small photonic crystal cavities
}

Wang, Fengwen; Christiansen, Rasmus Ellebæk; Yu, Yi; Mørk, Jesper; Sigmund, Ole

Published in:

Applied Physics Letters

Link to article, DOI:

$10.1063 / 1.5064468$

Publication date:

2018

Document Version

Publisher's PDF, also known as Version of record

Link back to DTU Orbit

Citation (APA):

Wang, F., Christiansen, R. E., Yu, Y., Mørk, J., \& Sigmund, O. (2018). Maximizing the quality factor to mode volume ratio for ultra-small photonic crystal cavities. Applied Physics Letters, 113(24), [241101]. https://doi.org/10.1063/1.5064468

\section{General rights}

Copyright and moral rights for the publications made accessible in the public portal are retained by the authors and/or other copyright owners and it is a condition of accessing publications that users recognise and abide by the legal requirements associated with these rights.

- Users may download and print one copy of any publication from the public portal for the purpose of private study or research.

- You may not further distribute the material or use it for any profit-making activity or commercial gain

- You may freely distribute the URL identifying the publication in the public portal 
Maximizing the quality factor to mode volume ratio for ultra-small photonic crystal cavities

Fengwen Wang, Rasmus Ellebæk Christiansen, Yi Yu, Jesper Mørk, and Ole Sigmund

Citation: Appl. Phys. Lett. 113, 241101 (2018); doi: 10.1063/1.5064468

View online: https://doi.org/10.1063/1.5064468

View Table of Contents: http://aip.scitation.org/toc/apl/113/24

Published by the American Institute of Physics

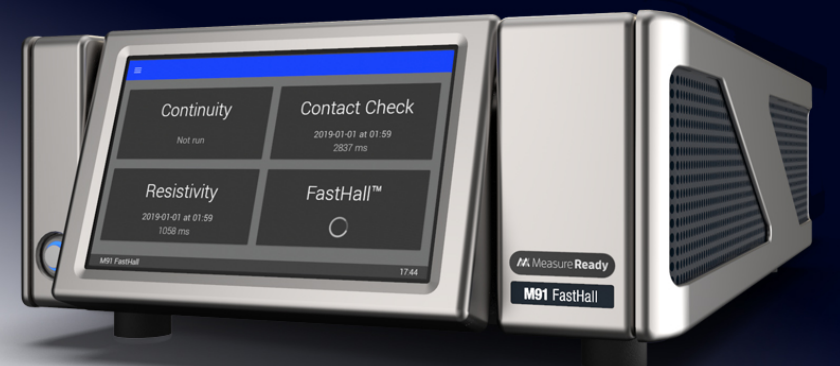

M Measure Ready M91 FastHall ${ }^{\mathrm{TM}}$ Controller

A revolutionary new instrument for complete Hall analysis 


\title{
Maximizing the quality factor to mode volume ratio for ultra-small photonic crystal cavities
}

\author{
Fengwen Wang, ${ }^{1, a)}$ Rasmus Ellebæk Christiansen, ${ }^{1} \mathrm{Yi} \mathrm{Yu}^{2}{ }^{2}$ Jesper Mørk, ${ }^{2}$ \\ and Ole Sigmund ${ }^{1}$ \\ ${ }^{1}$ Section of Solid Mechanics, Department of Mechanical Engineering, Technical University of Denmark, \\ 2800 Kgs. Lyngby, Denmark \\ ${ }^{2}$ DTU Fotonik, Department of Photonics Engineering, Technical University of Denmark, 2800 Kgs. Lyngby, \\ Denmark
}

(Received 4 October 2018; accepted 24 November 2018; published online 11 December 2018)

\begin{abstract}
Small manufacturing-tolerant photonic crystal cavities are systematically designed using topology optimization to enhance the ratio between the quality factor and mode volume, $Q / V$. For relaxed manufacturing tolerance, a cavity with a bow-tie shape is obtained which confines light beyond the diffraction limit into a deep-subwavelength volume. Imposition of a small manufacturing tolerance still results in efficient designs, however, with diffraction-limited confinement. Inspired by numerical results, an elliptic ring grating cavity concept is extracted via geometric fitting. Numerical evaluations demonstrate that for small sizes, topology-optimized cavities enhance the $Q / V$-ratio by up to two orders of magnitude relative to standard $L 1$ cavities and more than one order of magnitude relative to shape-optimized $L 1$ cavities. An increase in cavity size can enhance the $Q / V$-ratio by an increase in the $Q$-factor without a significant increase in $V$. Comparison between optimized and reference cavities illustrates that significant reduction of $V$ requires big topological changes in the cavity. Published by AIP Publishing. https://doi.org/10.1063/1.5064468
\end{abstract}

The strong light-matter interaction is key in a wide range of photonic and optoelectronic applications, including low threshold lasers, ${ }^{1-4}$ sensors, ${ }^{5}$ nonlinear optics, ${ }^{6}$ cavity quantum electrodynamics, ${ }^{7}$ switching, ${ }^{8,9}$ and optomechanics. ${ }^{10}$ In a cavity, the local photon density of states (LDOS) scales proportionally to the $Q / V$-ratio. An increase in LDOS in a cavity can lead to enhanced spontaneous emission through the Purcell effect. ${ }^{11}$ Both photonic crystal $(\mathrm{PhC})$ and plasmonic cavities have been used to enhance the Purcell effect. ${ }^{12-15}$ $\mathrm{PhC}$ cavities increase the temporal confinement of light in a material, as represented by their high $Q$, and are restricted by the diffraction-limited spatial confinement of the light, measured in terms of $V .^{12-14}$ Plasmonic cavities are capable of increasing the spatial confinement beyond the diffraction limit, i.e., a low $V$ can be attained, but are restricted to small $Q$-values due to ohmic losses. ${ }^{15}$ Miniaturization of cavities with a high $Q / V$-ratio is in demand to enhance the light-matter interaction and reduce footprint for compact integrated optical circuits.

Previously, many studies considered the design of dielectric $\mathrm{PhC}$ cavities with enhanced $Q$ while keeping a diffraction-limited $V \sim(\lambda / n)^{3}$. Most of the studies focused on shape optimization (SO) by changing locations or radii of air holes ${ }^{12-14}$ or using gradient-based geometry projection methods. ${ }^{16,17}$ These studies mainly focused on the conventional $\mathrm{Ln}$ or $H n$ cavities $^{12-14,16,17}$ ( $n$ is the number of filled holes in the $\mathrm{PhC}$ ) and assumed very large in-plane dimensions and thus large footprint to reduce in-plane loss. Recently, bow-tie shaped $\mathrm{PhC}$ cavities consisting of two tip-to-tip opposite components were studied. ${ }^{18-21}$ However, the low $V$ in these cavities is found to be highly dependent on the features

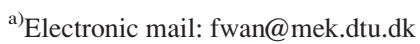

between the two tips and hence extremely sensitive to manufacturing variations. 3D electron beam lithography (EBL) can fabricate $\mathrm{PhC}$ structures with hole size down to about $40 \mathrm{~nm},{ }^{22}$ and more recently, the width of the bow-tie tip connection has been controlled to $12 \mathrm{~nm}$ with an error region of $\pm 5 \mathrm{~nm}^{23}$ The fabrication accuracy puts a limit on the resolution of the tip-region, which is important to take into account when optimizing the design.

A density-based topology optimization (TO) method was also employed to design finite-size $\mathrm{PhC}$ cavities with an enhanced $Q / V$-ratio. ${ }^{24}$ Even though these optimized $\mathrm{PhC}$ cavities exhibit strongly enhanced performance, they are difficult to manufacture, as they contain prohibitively small holes or holes with irregular patterns and sharp features. Such features, largely determined by the underlying mesh resolution, may also result in large modelling errors and erroneous performance estimates. In this study, we employ TO with manufacturing and length-scale control to systematically design manufacturable 3D PhC membrane cavities, demonstrating an increase in the $Q / V$-ratio by up to two orders of magnitude relative to the standard $L 1$ cavity contained within a square membrane (see Fig. 1) and more than one order of magnitude relative to a SO $L 1$ cavity. In addition, we investigate the influence of the cavity size on the performance of the optimized and reference cavities.

In the limit of a low-loss cavity, the $Q / V$-ratio is only an approximation to the LDOS dominated by the contribution of a single resonant mode. ${ }^{11,24}$ The $Q / V$-ratio also describes the spontaneous emission enhancement in the case where the emitter linewidth is narrower than the cavity linewidth. ${ }^{25}$ Hence, to directly target the goal of achieving a strong lightmatter interaction, the design goal is recast as the problem of maximizing a frequency-averaged LDOS in the $\mathrm{PhC}$ 


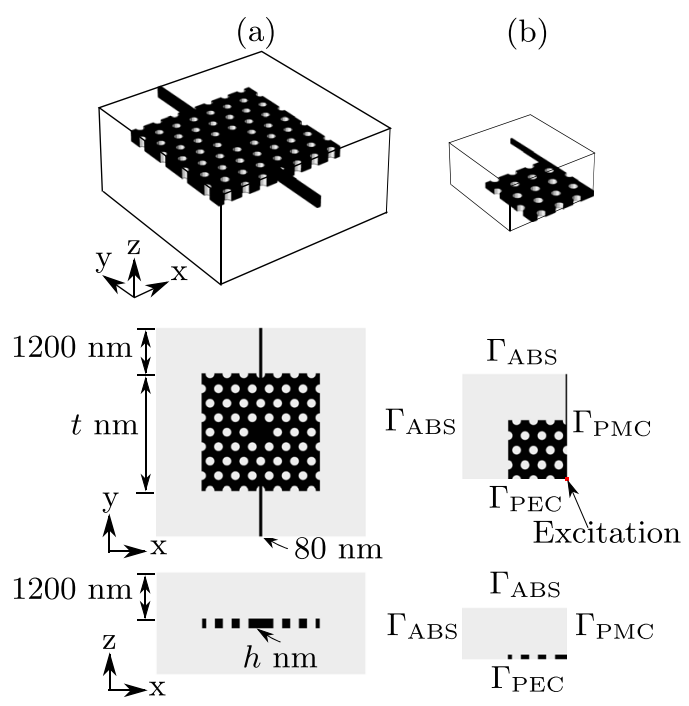

FIG. 1. Illustration of a 3D PhC membrane and the corresponding computational domain. (a) 3D PhC membrane. (b) Computational domain and boundary conditions.

membrane excited by a dipole. ${ }^{24}$ Using complex analysis, it can be shown that the LDOS may be calculated from the electric field using a simple multi-pole evaluation instead of a full frequency domain integration. ${ }^{24}$ By introducing a squared Lorentzian weight function, this evaluation is reduced to a single complex frequency evaluation of the electric field to obtain the LDOS for a given material configuration. The width of the weight function is gradually reduced over the course of the optimization using a continuation scheme to attain the final design for the targeted frequency. ${ }^{24}$

The cavity design and evaluation take offset in the $\mathrm{PhC}$ membrane illustrated in Fig. 1 with a finite size of $t \times t \times h$. The lattice constant is set to $a=440 \mathrm{~nm}$, and the membrane thickness is set to $h / a=0.568$. To avoid a free floating membrane, a thin beam of width $80 \mathrm{~nm}$ is attached to the membrane as shown in the top figure in Fig. 1(a). The dielectric material constituting the membrane is Indium Phosphide (InP) with a refractive index of $n_{\mathrm{InP}}=3.17$. The membrane is surrounded by air $\left(n_{\text {air }}=1\right)$ in all directions. Due to the imposition of symmetry, only one eighth of the full domain is modelled with appropriate boundary conditions as illustrated in Fig. 1(b), where $\Gamma_{\mathrm{PMC}}$ and $\Gamma_{\mathrm{PEC}}$ denote a perfect magnetic conductor (PMC, $\boldsymbol{n} \times \boldsymbol{H}=\mathbf{0}$ ) and a perfect electric conductor (PEC, $\boldsymbol{n} \times \boldsymbol{E}=\mathbf{0}$ ), respectively. Absorbing boundary conditions, $\Gamma_{\mathrm{ABS}}$, are used to truncate the computational domain. The membrane is excited by a dipole oriented along the $\mathrm{x}$-direction with an angular frequency, $\omega$, modelled as a current $\boldsymbol{J}(\boldsymbol{x}) \sim \boldsymbol{e}_{x} e^{i \omega t} \delta\left(\boldsymbol{x}-\boldsymbol{x}_{0}\right)$. The total electric field is governed by

$$
\nabla \times \frac{1}{\mu(\boldsymbol{x})} \nabla \times \boldsymbol{E}(\boldsymbol{x})-\varepsilon(\boldsymbol{x}) \omega^{2} \boldsymbol{E}(\boldsymbol{x})=i \omega \boldsymbol{J}(\boldsymbol{x}) .
$$

The finite element method is employed to solve the scattering problem in Eq. (1). The solver is implemented using the parallel sparse-matrix library PETSc ${ }^{26}$ and the MUltifrontal Massively Parallel sparse direct Solver (MUMPS). ${ }^{27}$

Based on the finite element discretization, a continuous design variable, $\rho \in[0,1]$, is introduced in each element to represent the material occupation in the element, and the elemental refractive index is interpolated using ${ }^{28}$

$$
n(\rho)=\rho\left(n_{\text {InP }}-n_{\text {Air }}\right)+n_{\text {Air }} .
$$

The design is iteratively updated using the gradient-based optimization method, the Method of Moving Asymptotes (MMA). ${ }^{29}$ The sensitivities of the objective and constraints are derived using adjoint sensitivity analysis. ${ }^{24,30}$ The software used to design cavities including the finite element solver and MMA implementation is developed in-house.

In order to verify the performance of the optimized cavities, they are imported into the commercial software COMSOL Multiphysics 5.3 and evaluated using eigenvalue analysis. ${ }^{31}$ The TO designs are extracted from the FEM model by performing an isometric mapping and extracting the design for the isovalue of 0.5 in a post processing step. This avoids stair-casing caused by the FEM mesh of the optimization model which in turn would limit accurate production.

As the first optimization case, we explore the possible performance attained by TO cavities. Hence, we design a free floating PhC cavity with a size of $t / a=7$, allowing small and sharp features. The target wavelength is $\lambda=1550 \mathrm{~nm}$. As the initial guess for the optimization, we use the reference $L 1$ cavity shown in Fig. 1 consisting of a hexagonal lattice with an air cylinder radius of $r=0.26 a$ without the beam in the center region. The full $\mathrm{PhC}$ region is freely designable. The center of the optimized cavity shown in Fig. 2(a) resembles a bow-tie with a tip gap of $10 \mathrm{~nm}$ at the cavity center, which is surrounded by a ring-like grating. As expected, small features are observed in the optimized cavity, which are likely impossible to fabricate accurately using present day techniques.

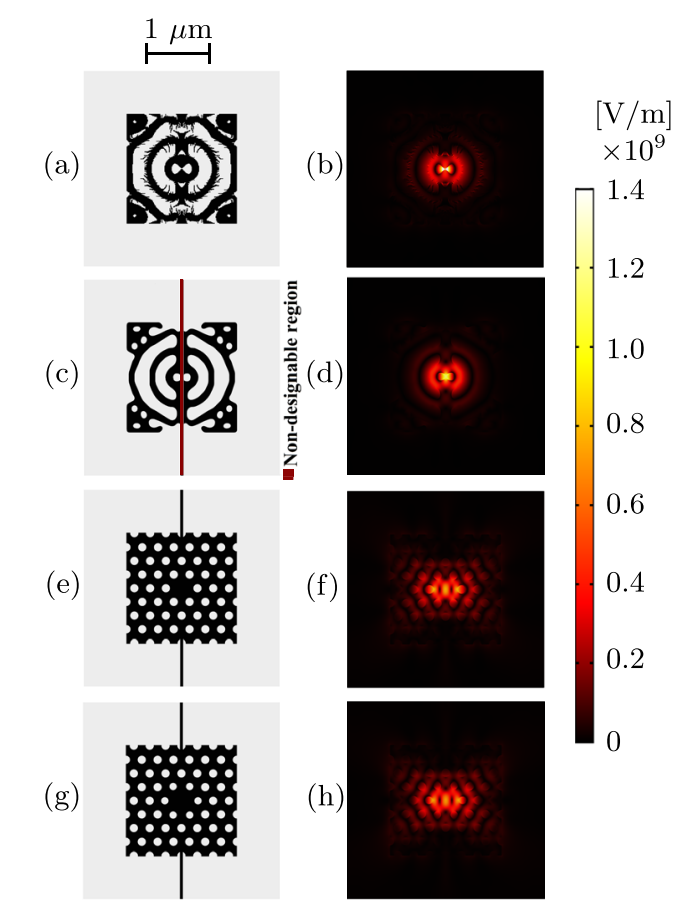

FIG. 2. Left column: TO cavities and reference ones with $t / a=7$. Right column: electric field norm $\|\boldsymbol{E}\|$ of the corresponding fundamental resonant modes. (a) and (b) TO 3D PhC membrane cavity with small features. (c) and (d) TO 3D PhC membrane cavity with a minimum length scale of $74 \mathrm{~nm}$. (e) and (f) $L 1$ cavity. (g) and (h) SO $L 1$ cavity. 
To avoid the small features present in the previous design, a minimum length scale is introduced in the design by applying a smoothened Heaviside projection filtering technique to the design field. ${ }^{32}$ The smallest feature that can be fabricated is determined mainly by the EBL and the semiconductor dry etching. As for our InP platform, we found that it is preferable to keep this value larger than $50 \mathrm{~nm}$; otherwise, it will be difficult to make it etched through. Here, a filter radius of $96 \mathrm{~nm}$ is employed, and a minimum length scale of $74 \mathrm{~nm}$ is enforced in both dielectric material and air using a geometrical constraint approach. ${ }^{33}$ Furthermore, an $80 \mathrm{~nm}$ wide non-designable region occupied by $\mathrm{InP}$ is introduced at the center of the cavity, highlighted in the red in the optimized design in Fig. 2(c), as the mode should be confined inside the solid and not the air region. This has the effect that the size of the bow-tie region is changed. It is seen that all the features in the design are smooth and conform to the imposed length scale. The smoothened bow-tie is surrounded by a smooth elliptic ring grating and (less important) corner PhC-like regions.

To evaluate the performance of the optimized cavities, we use the standard $L 1$ cavity shown in Fig. 1(a) [also shown in Fig. 2(e)] as a reference ( $L 1$ has an antinode of $\|\boldsymbol{E}\|$ at the cavity center, while $H 0$ has an node $\left.{ }^{14}\right)$. As an additional reference, we use a SO $L 1$ cavity obtained using a parameter sweep over the hole radius $\left(r_{0}\right)$ and lattice constant $\left(a_{0}\right)$ of the air cylinders closest to the cavity center, with $r_{0} \in$ $[0.22 a, 0.32 a]$ and $a_{0} \in[220,500] \mathrm{nm}$. The SO $L 1$ cavity with the largest $Q / V$-ratio is obtained for $r_{0}=0.23 a$ and $a_{0}=460 \mathrm{~nm}$ and is shown in Fig. $2(\mathrm{~g})$.

The normalized electric field norm of the resonant modes in the optimized and reference cavities is shown in the right column of Fig. 2. The performance of the cavities is summarized in Table I. It is seen in Fig. 2(b) that the cavity with small features exhibits an extremely high field intensity at its center. Hence, the optimized cavity can concentrate light into a deep-subwavelength volume with $V=0.00026(\lambda / n)^{3}$. Here, $n$ is the refractive index of the cavity material. The mode volume is calculated using ${ }^{20} \quad V=\frac{\int \varepsilon(\boldsymbol{x})|\boldsymbol{E}(\boldsymbol{x})|^{2} d x}{\max \left\{\varepsilon(\boldsymbol{x})|\boldsymbol{E}(\boldsymbol{x})|^{2}\right\}}$. Moreover, the surrounding grating structure adapted to the bow-tie shape further reduces field intensity away from the cavity center, which leads to an increase in $Q$ and a further reduced $V$ for the targeted resonant mode. Hence, the optimized cavity shown in Fig. 2(a) displays at least two order of magnitude lower $V$ than the optimized $H 0$ cavity by Wang et al. ${ }^{17}$ and five time smaller $V$ than the other proposed cavities by Gondarenko and Lipson. ${ }^{18}$ Furthermore, it possesses a high $Q / V$-ratio, which is at least two times that of the highest $Q / V$-ratio obtained for a dielectric bow-tie cavity proposed by

TABLE I. Performance of the TO, $L 1$, and SO $L 1$ cavities with $t / a=7$ for the fundamental resonant mode.

\begin{tabular}{lcccc}
\hline \hline Design & $\lambda(\mathrm{nm})$ & $Q$ & $V\left[(\lambda / n)^{3}\right]$ & $Q / V\left[(n / \lambda)^{3}\right]$ \\
\hline TO (a) & 1549 & 1062 & 0.0002651 & $4006 \times 10^{3}$ \\
TO Len (c) & 1554 & 2979 & 0.1083 & $27.50 \times 10^{3}$ \\
$L 1(\mathrm{e})$ & 1451 & 265.4 & 0.3142 & $0.8446 \times 10^{3}$ \\
SO $L 1(\mathrm{~g})$ & 1506 & 486.7 & 0.3330 & $1.462 \times 10^{3}$ \\
\hline \hline
\end{tabular}

Lu et al. ${ }^{19}$ and a hybrid photonic-plasmonic nanobeam cavity at room temperature by Conteduca et al. ${ }^{34}$ It is known that $V$ is mainly determined by the size of the gap in the bow-tie shape at the cavity center. The smaller the gap, the smaller the $V .{ }^{19}$ In this work, the numerical resolution and post processing limited the gap size between two bow-tie tips to $10 \mathrm{~nm}$.

The field intensity at the cavity center for the design with restricted spatial resolution, shown in Figs. 2(c) and 2(d), is lower than for the previous design shown in Figs. 2(a) and 2(b). However, it is still significantly larger than the $L 1$ and SO $L 1$ cavities shown in Figs. 2(e)-2(h). Moreover, the spatial confinement of the field pattern is smaller than in the reference cavities. Hence, it is evident that the optimized cavity possesses a significantly enhanced $Q / V$-ratio relative to both reference cavities (see Table I). However, the introduction of the non-design domain at the cavity center and the enforcement of the length scale prohibit the appearance of small features, which in turn restricts the optimized design from attaining a $V$ below the diffraction limit of $V \sim 0.1(\lambda / n)^{3}$. It is observed that the TO cavity with small features confines light in the air regions, while the one with the imposed length scale confines light inside InP. The non-design domain guarantees the confinement of light in the material region at the cavity center, which is important for applications. ${ }^{3}$

Compared to the standard $L 1$ cavity, the SO $L 1$ cavity enhances the $Q / V$-ratio by an increased $Q$ with a small increase in $V$. The TO cavity with the imposed length scale enhances the $Q / V$-ratio by both increased $Q$ and reduced $V$ and displays a $Q / V$-ratio at least 18 times higher than both references. The finite size of the $\mathrm{PhC}$ membrane limits the $Q$-value due to in-plane loss. The elliptic Bragg gratings created in the TO cavities reduce in-plane loss, hereby facilitating a significant increase in $Q$.

Next, we study the effect of the outer dimensions of the $\mathrm{PhC}$ cavity on the attainable $Q / V$-ratio.

TO cavities, designed with resolution limitation and with different maximum outer dimensions of $t / a=4.273, t / a$ $=7, t / a=11$, and $t / a=15$, are presented in Figs. 3(a) $-3(\mathrm{~d})$.
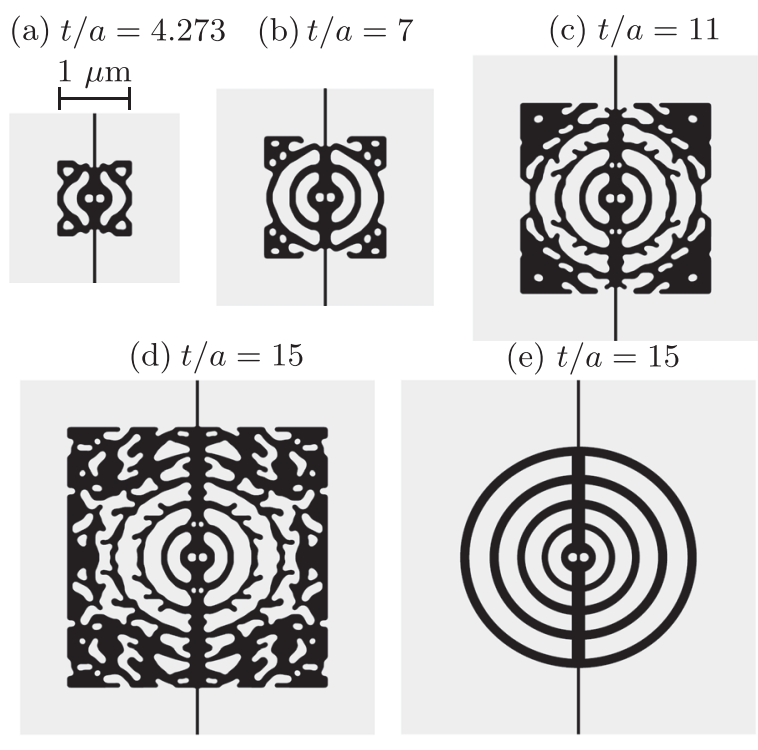

FIG. 3. (a)-(d) TO 3D PhC membrane cavities with different sizes. (e) Extracted elliptic ring grating cavity. 
It is seen that all the optimized cavities retain the bow-tie shape region surrounded by the elliptic ring gratings adapted to a central bow-tie shape, indicating the importance of both types of features in attaining a high $Q / V$-ratio. More complex geometric features are seen to appear in the largest cavity [Fig. 3(d)]. These intricate features are however less important for the design performance due to the low field intensity further from the cavity center.

By extracting the elliptic rings and bow-tie feature from the TO cavity in Fig. 3(d) and fitting a simplified elliptic grating structure with varying bar widths and spacings, an elliptic ring grating cavity is obtained [see Fig. 3(e)]. The corresponding elliptic ring grating cavities for $t / a=11, t / a$ $=7, t / a=4.273$ are obtained by removing one, two, and three ellipse rings from the design in Fig. 3(e).

The performances of the reference, TO with resolution limitation, and parametrized ring cavities are shown in Fig. 4. SO $L 1$ cavities are obtained by performing a $\mathrm{SO}$ for each individual size. Note that the sizes of the elliptic ring grating cavities deviate from the other cavities. The $t / a$-values reported in Fig. 4 correspond to the dimension in the $x$-direction. As expected, the larger the cavity size, the larger the $Q / V$ ratio. Unsurprisingly, the TO cavities are seen to exhibit the best performance followed by the elliptic ring grating cavities. The elliptic ring grating cavities possess smaller $Q$ compared to the corresponding TO cavities but have similar $V$. Moreover, it is seen that increasing the cavity size can enhance $Q$ significantly; however, $V$ does not change much since the main contribution to the low $V$ stems from the bow-tie at the cavity center. For the largest size, there is no significant difference in terms of $Q$ between the TO and SO cavities since the in-plane loss is very small in the large cavity and the $Q$ is mainly dominated by out-of-plane loss. Comparing the TO designs to the $L 1$ and SO $L 1$ cavities, a
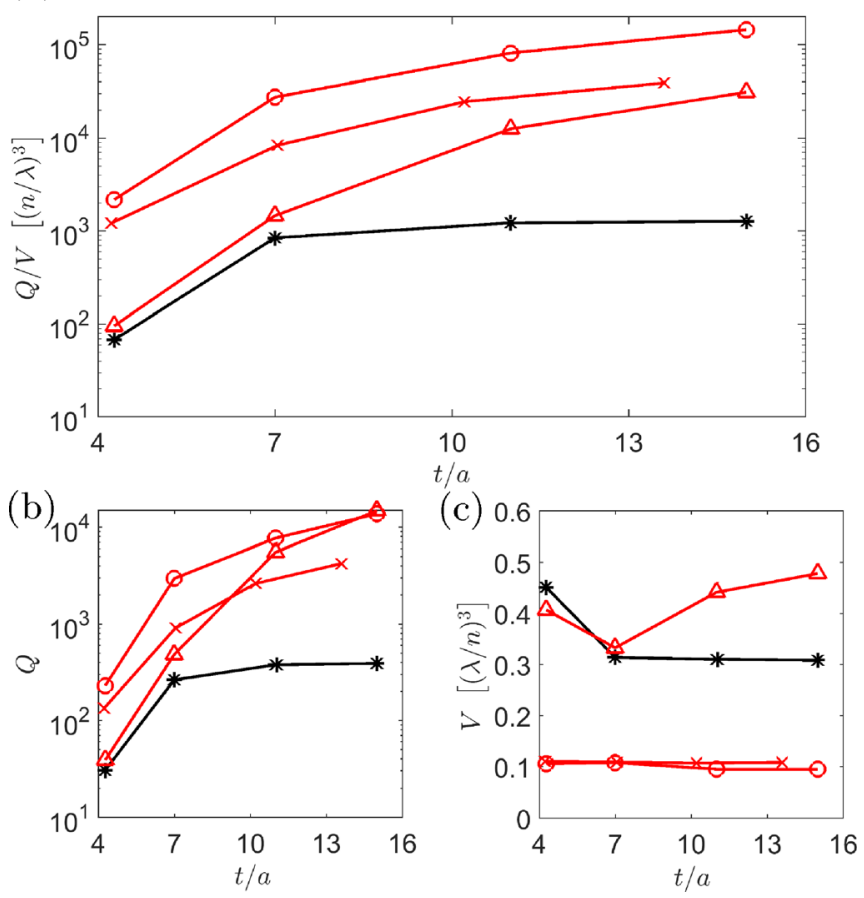

FIG. 4. Overall performance comparison of different cavities vs cavity size. (a) $Q / V$ vs cavity size. (b) $Q$ vs cavity size. (c) $V$ vs cavity size. significant decrease in $V$ is observed for all sizes. This indicates that to attain a lower $V$, significant geometric changes to the cavity are required. Moreover, the extracted elliptic ring grating cavities exhibit more in-plane losses represented by smaller $Q$ than the SO $L 1$ and TO cavities for large cavity size. This implies that the smaller branches attached in the ring gratings and the features at the membrane corners in Figs. 3(c) and 3(d) can facilitate a higher $Q$. However, the simplicity of the parametrized elliptic ring cavity may be appealing from a fabrication point of view.

In conclusion, a density-based TO method has been employed to the systematic design of manufacturable small finite-size membrane cavities with a smallest feature size of $74 \mathrm{~nm}$, well above standard EBL tolerances. ${ }^{22}$ Furthermore, the influence of cavity size on $Q$ and $V$ was investigated. Inspired by the TO designs, elliptic ring grating cavities were extracted using parametric models. The overall comparison of cavity performances demonstrates that the TO cavities perform best among all the cavities considered followed by the extracted ring grating cavities. By increasing the cavity size, one can obtain higher $Q$; however, there is no significant improvement in $V$. The lower $V$ requires significant geometric changes in the cavity center, such as from $L 1$ to elliptic ring grating cavities. Moreover, resolution restrictions lead to near-diffraction-limited volumes. Smaller features are required to reach a deep-subwavelength mode volume. By reducing the minimum length scale and introducing a smaller fixed material region at the cavity center, it would be possible to obtain designs with significantly lower $V$ while ensuring that the field is confined to the solid. Even though this study is mainly focused on designing cavities with field confinement in solid, the whole procedure can be used to design cavities with field confinement in the air as well, suitable for other applications such as optical tweezers. ${ }^{35}$

This work was financially supported by Villum Fonden via the NATEC Center of Excellence (Grant No. 8692).

${ }^{1}$ O. Painter, R. Lee, A. Scherer, A. Yariv, J. O'brien, P. Dapkus, and I. Kim, "Two-dimensional photonic band-gap defect mode laser," Science 284, 1819-1821 (1999).

${ }^{2}$ Y. Ota, K. Watanabe, S. Iwamoto, and Y. Arakawa, "Nanocavity-based self-frequency conversion laser," Opt. Express 21, 19778-19789 (2013).

${ }^{3}$ S. Matsuo, T. Sato, K. Takeda, A. Shinya, K. Nozaki, H. Taniyama, M. Notomi, K. Hasebe, and T. Kakitsuka, "Ultralow operating energy electrically driven photonic crystal lasers," IEEE J. Sel. Top. Quantum Electron. 19, 4900311-4900311 (2013).

${ }^{4}$ W. Xue, Y. Yu, L. Ottaviano, Y. Chen, E. Semenova, K. Yvind, and J. Mørk, "Threshold characteristics of slow-light photonic crystal lasers," Phys. Rev. Lett. 116, 063901 (2016).

${ }^{5}$ G. Pitruzzello and T. F. Krauss, "Photonic crystal resonances for sensing and imaging," J. Opt. 20, 073004 (2018).

${ }^{6} \mathrm{M}$. Soljacic and J. D. Joannopoulos, "Enhancement of nonlinear effects using photonic crystals," Nat. Mater. 3, 211-219 (2004).

${ }^{7}$ T. Tiecke, J. D. Thompson, N. P. de Leon, L. Liu, V. Vuletić, and M. D. Lukin, "Nanophotonic quantum phase switch with a single atom," Nature 508, 241 (2014).

${ }^{8}$ C. Husko, A. De Rossi, S. Combrié, Q. V. Tran, F. Raineri, and C. W. Wong, "Ultrafast all-optical modulation in GaAs photonic crystal cavities," Appl. Phys. Lett. 94, 021111 (2009).

${ }^{9}$ Y. Yu, Y. Chen, H. Hu, W. Xue, K. Yvind, and J. Mørk, "Nonreciprocal transmission in a nonlinear photonic crystal Fano structure with broken symmetry," Laser Photonics Rev. 9, 241-247 (2015). 
${ }^{10}$ T. J. Kippenberg and K. J. Vahala, "Cavity optomechanics: Back-action at the mesoscale," Science 321, 1172-1176 (2008).

${ }^{11}$ E. M. Purcell, "Spontaneous emission probabilities at radio frequencies," Phys. Rev. 69, 681 (1946).

${ }^{12}$ Y. Akahane, T. Asano, B.-S. Song, and S. Noda, "High-Q photonic nanocavity in a two-dimensional photonic crystal," Nature 425, 944 (2003).

${ }^{13}$ U. P. Dharanipathy, M. Minkov, M. Tonin, V. Savona, and R. Houdr, "High-Q silicon photonic crystal cavity for enhanced optical nonlinearities,” Appl. Phys. Lett. 105, 101101 (2014).

${ }^{14}$ M. Minkov and V. Savona, "Automated optimization of photonic crystal slab cavities," Sci. Rep. 4, 5124 (2014).

${ }^{15} \mathrm{~J}$. B. Khurgin, "How to deal with the loss in plasmonics and metamaterials," Nat. Nanotechnol. 10, 2 (2015).

${ }^{16}$ W. R. Frei, H. Johnson, and K. D. Choquette, "Optimization of a single defect photonic crystal laser cavity," J. Appl. Phys. 103, 033102 (2008).

${ }^{17}$ D. Wang, Z. Yu, Y. Liu, X. Guo, C. Shu, S. Zhou, and J. Zhang, "Ultrasmall modal volume and high Q factor optimization of a photonic crystal slab cavity,” J. Opt. 15, 125102 (2013).

${ }^{18}$ A. Gondarenko and M. Lipson, "Low modal volume dipole-like dielectric slab resonator," Opt. Express 16, 17689-17694 (2008).

${ }^{19}$ Q. Lu, F.-J. Shu, and C.-L. Zou, "Dielectric bow-tie nanocavity," Opt. Lett. 38, 5311-5314 (2013)

${ }^{20} \mathrm{~S}$. Hu and S. M. Weiss, "Design of photonic crystal cavities for extreme light concentration," ACS Photonics 3, 1647-1653 (2016).

${ }^{21}$ H. Choi, M. Heuck, and D. Englund, "Self-similar nanocavity design with ultrasmall mode volume for single-photon nonlinearities," Phys. Rev. Lett. 118, 223605 (2017).

${ }^{22} \mathrm{~K}$. Yamazaki and H. Yamaguchi, "Renovation of three-dimensional electron beam lithography for improvement of positioning accuracy and reduction of turnaround time," Jpn. J. Appl. Phys. Part 1 54, 06FD02 (2015).

${ }^{23}$ S. Hu, M. Khater, R. Salas-Montiel, E. Kratschmer, S. Engelmann, W. M Green, and S. M. Weiss, "Experimental realization of deep-subwavelength confinement in dielectric optical resonators," Sci. Adv. 4, eaat2355 (2018).

${ }^{24}$ X. Liang and S. G. Johnson, "Formulation for scalable optimization of microcavities via the frequency-averaged local density of states," Opt. Express 21, 30812-30841 (2013).
${ }^{25}$ J. Mork and G. Lippi, "Rate equation description of quantum noise in nanolasers with few emitters," Appl. Phys. Lett. 112, 141103 (2018).

${ }^{26}$ S. Balay, S. Abhyankar, M. F. Adams, J. Brown, P. Brune, K. Buschelman, L. Dalcin, V. Eijkhout, W. D. Gropp, D. Kaushik, M. G. Knepley, D. A. May, L. C. McInnes, R. T. Mills, T. Munson, K. Rupp, P. Sanan, B. F. Smith, S. Zampini, H. Zhang, and H. Zhang, "PETSc users manual," Technical Report ANL-95/11-Revision 3.9 (Argonne National Laboratory, 2018).

${ }^{27}$ P. R. Amestoy, I. S. Duff, J. Koster, and J.-Y. L'Excellent, “A fully asynchronous multifrontal solver using distributed dynamic scheduling," SIAM J. Matrix Anal. Appl. 23, 15-41 (2001).

${ }^{28}$ R. E. Christiansen, J. Vester-Petersen, S. P. Madsen, and O. Sigmund, "A non-linear material interpolation for design of metallic nano-particles using topology optimization," Comput. Methods Appl. Mech. Eng. 343, 23-39 (2019).

${ }^{29} \mathrm{~K}$. Svanberg, "The method of moving asymptotes - a new method for structural optimization," Int. J. Numer. Methods Eng. 24, 359-373 (1987).

${ }^{30}$ J. S. Jensen and O. Sigmund, "Topology optimization for nano-photonics," Laser Photonics Rev. 5, 308-321 (2011).

${ }^{31}$ J. R. de Lasson, L. H. Frandsen, P. Gutsche, S. Burger, O. S. Kim, O. Breinbjerg, A. Ivinskaya, F. Wang, O. Sigmund, T. Häyrynen, J. Mørk, and N. Gregersen, "Benchmarking five numerical simulation techniques for computing resonance wavelengths and quality factors in photonic crystal membrane line defect cavities," Opt. Express 26, 11366-11392 (2018)

${ }^{32}$ F. Wang, B. S. Lazarov, and O. Sigmund, "On projection methods, convergence and robust formulations in topology optimization," Struct. Multidiscip. Optim. 43, 767-784 (2011).

${ }^{33}$ M. Zhou, B. S. Lazarov, F. Wang, and O. Sigmund, "Minimum length scale in topology optimization by geometric constraints," Comput. Methods Appl. Mech. Eng. 293, 266-282 (2015).

${ }^{34}$ D. Conteduca, C. Reardon, M. G. Scullion, F. DellOlio, M. N. Armenise, T. F. Krauss, and C. Ciminelli, "Ultra-high Q/V hybrid cavity for strong light-matter interaction,” APL Photonics 2, 086101 (2017).

${ }^{35}$ M. L. Juan, M. Righini, and R. Quidant, "Plasmon nano-optical tweezers," Nat. Photonics 5, 349-356 (2011). 